\title{
A review on swine flu infection and strategies for its treatment in future
}

\begin{abstract}
H1N1 influenza infection is one of the most influential types of influenza viruses that enamored and surrenders almost all age gatherings of human populaces. This paper exhibits nitty-gritty data in regards to general mechanism of $\mathrm{H}_{1} \mathrm{~N}_{1}$ infection, the impact of swine flu in people, correlation of swine flu infection with liquor utilization, common cold, and noteworthiness of obesity. Pandemic spread in 2009, the pharmacological role of plant supplements in context of $\mathrm{H}_{1} \mathrm{~N}_{1}$ and their uses and a metric study showing the ranking of supplements with the highest frequency of occurrence (F supplement) and ranking of supplement with highest probabilities of co-occurrence with $\mathrm{H}_{1} \mathrm{~N}_{1}$ (P supplement) has been portrayed in the present review.
\end{abstract}

Keywords: $\mathrm{H}_{1} \mathrm{~N}_{1}$, influenza virus, swine flu, pandemic, f supplement, p supplement
Volume 9 Issue I - 2020

\author{
Prashant Kumar Dhakad, Mahaveer Singh \\ School of Pharmaceutical Sciences, Jaipur National University, \\ India
}

Correspondence: Prashant Kumar Dhakad PhD, Assistant Professor, School of Pharmaceutical Sciences, Jaipur National University, Jagatpura, Jaipur, Rajasthan-302017, India, Email dhakadprashant654@gmail.com

Received: November 22, 2019 | Published: January 24, 2020

\section{Introduction}

The present survey deals with information regarding the sufferings caused during the $\mathrm{H}_{1} \mathrm{~N}_{1}$ infection happened in 2009, pathophysiology of swine influenza disease, genomic impact of swine influenza in people, impact of way of life factors including liquor, normal cold, and weight on swine influenza infection. The present review is valuable for the human services suppliers, patients; individuals at high hazard including kids, pregnant ladies and those with certain ailments and general public for mindfulness and taking preventive measures. Manifestations of the H1N1 influenza incorporate fever, chills, queasiness, regurgitating, body hurts, laziness and weariness, which generally show up in quick progression. The most widely recognized reason for death from the infection is respiratory failure, yet different reasons for mortality incorporate sepsis, lack of hydration and electrolyte awkwardness. Pneumonia is the most widely recognized entanglement of regular flu. Natural, nourishing and allopathic treatment measures have likewise been talked about in the present survey.

$\mathrm{H}_{1} \mathrm{~N}_{1}$ influenza is otherwise called swine influenza on the grounds that previously, the individuals who got it had direct contact with pigs. That changed quite a long while prior, when another infection raised that spread among individuals who hadn't been close to pigs. In $2009, \mathrm{H}_{1} \mathrm{~N}_{1}$ was spreading quickly around the globe, so the World Health Organization considered it a pandemic. From that point forward, individuals have kept on becoming ill from swine influenza, yet not the same number of. The April 2009 swine influenza flareup (pandemic) was because of disease with the $\mathrm{H}_{1} \mathrm{~N}_{1}$ infection and was first seen in Mexico. $\mathrm{H}_{1} \mathrm{~N}_{1}$ (Swine influenza) is a viral disease brought about by flu A subtype infection of Orthomyxoviridae family. It has single stranded negative sense portioned RNA genome which codes for 16 distinct proteins. The state of infection might be circular or filamentous having size of $80-120 \mathrm{~nm}$. Nucleocapsid of infection has symmetric helical structure and its surface has glycoprotein hemagglutinin (HA) and compound neuraminidase (NA).

HA helps in connection of infection through sialic corrosive of glycoprotein on the outside of upper respiratory tract or erythrocytes of host and chemical NA cuts sialic corrosive from cell surface and free relatives of infection from tainted patient cells. Swine influenza taints the respiratory tract of pigs, bringing about nasal discharges, a yelping hack, diminished hunger, and drowsy conduct. Swine influenza delivers the majority of indistinguishable side effects in pigs from human influenza create in individuals. Swine influenza can last around one to about fourteen days in pigs that endure. In various occurrences, individuals have built up the swine influenza disease when they are intently connected with pigs (for instance, ranchers, pork processors), and moreover, pig populaces have every so often been contaminated with the human influenza contamination. In many cases, the crossspecies diseases (swine-source infection to man; human influenza infection to pigs) have stayed in neighborhoods have not caused national or overall contaminations in either pigs or people. Shockingly, this cross-species circumstance with flu infections (human diseases with swine infections) has could possibly change. Agents chose the 2009 purported "swine influenza" strain, first found in Mexico, ought to be named novel $\mathrm{H}_{1} \mathrm{~N}_{1}$ influenza since it was predominantly found contaminating individuals and displays two primary surface antigens, $\mathrm{H}_{1}$ (hemagglutinin type 1) and $\mathrm{N}_{1}$ (neuraminidase type1). The eight RNA strands from novel $\mathrm{H}_{1} \mathrm{~N}_{1}$ influenza have one strand gotten from human influenza strains, two from avian (winged creature) strains, and five from swine strains. The principle swine influenza infections in pigs in the ongoing years are swine triple reassortant (tr; it implies a viral strain with qualities from three distinct life forms) $\mathrm{H}_{1} \mathrm{~N}_{1}, \operatorname{trH}_{3} \mathrm{~N}_{2}$, and $\operatorname{trH}_{1} \mathrm{~N}_{2}$. Be that as it may, in August 2018, China previously revealed another swine influenza flare-up in pigs in Liaoning area. The pig influenza strain known as African swine influenza (albeit a few scientists think it began in Russia), practically $100 \%$ deadly to pigs, was the reason. This strain is profoundly irresistible, gets by in warmth and cold conditions, and can stay feasible and irresistible on surfaces for a considerable length of time to weeks. As of now, there is no successful antibody or medication to stop its spread, so the illness is treated by quick butcher of contaminated pigs. China has about half of the world's pig populace and depends on pork to give a lot of protein for the Chinese populace. ${ }^{1-3}$ 


\section{Swine flu infecting humans}

Numerous specialists presently think about that two principle arrangements of occasions can prompt swine influenza turning into a noteworthy reason for flu sickness in people. To start with, the flu infections (types A, B, C) are wrapped RNA infections with a portioned genome; this implies the viral RNA hereditary code is anything but a solitary strand of RNA yet exists as eight diverse RNA sections in the flu infections. A human (or winged creature) flu infection can contaminate a pig respiratory cell simultaneously as a swine flu infection; a portion of the reproducing RNA strands from the human infection can get erroneously encased inside the wrapped swine flu infection. For instance, one cell could contain eight swine influenza and eight human influenza RNA portions. Different blends of RNA fragments can bring about another subtype of infection (this procedure is known as antigenic move) that may be able to specially taint people yet at the same time show attributes remarkable to the swine flu infection. It is even conceivable to incorporate RNA strands from fowls, swine, and human flu infections into one infection if a solitary cell ends up tainted with each of the three sorts of flu (for instance, two winged creature influenza, three swine influenza, and three human influenza RNA portions to deliver a practical eightfragment new kind of influenza viral genome).

Little hereditary changes can aggregate after some time to deliver enough minor changes that in total adjust the infection's cosmetics over the long run (normally years). Pigs can assume an interesting job as a delegate host to new influenza types since pig respiratory cells can be contaminated straightforwardly with fowl, human, and other mammalian influenza infections. Therefore, pig respiratory cells can be contaminated with numerous sorts of influenza and can work as a "blending pot" for influenza RNA fragments. Flying creature's influenza infections, which more often than not taint the gastrointestinal cells of many fowl species, are shed in flying creature dung. Pigs can lift these infections up from the earth, and this is by all accounts the significant way that flying creature influenza infection RNA fragments enter the mammalian influenza infection populace. Swine influenza infections don't typically contaminate people. In any case, sporadic human contaminations with flu infections that ordinarily flow in swine and not individuals have happened. At the point when this occurs, these infections are classified "variation infections." They additionally can be indicated by including the letter "v" as far as possible of the infection subtype assignment. Human diseases with $\mathrm{H}_{1} \mathrm{~N}_{1} \mathrm{v}, \mathrm{H}_{3} \mathrm{~N}_{2} \mathrm{v}$ and $\mathrm{H}_{1} \mathrm{~N}_{2} \mathrm{v}$ infections have been recognized in the United States. ${ }^{4}$

Most ordinarily, human contaminations with variation infections happen in individuals with presentation to tainted pigs (e.g., kids close to pigs at a reasonable or laborers in the swine business). This is thought to happen for the most part when a tainted pig hacks or wheezes and beads with flu infection in them spread through the air. On the off chance that these beads land in your nose or mouth, or are breathed in, you can be contaminated. There likewise is some proof that you may get tainted by contacting something that has infection on it and afterward contacting your very own mouth or nose. A third method to perhaps get contaminated is to breathe in particles containing flu infection. Researchers aren't generally certain which of these methods for spread is the most well-known. Swine flu has not been demonstrated to be transmissible to individuals through eating appropriately took care of and arranged pork (pig meat) or different items got from pigs. In 2012, for instance, of 309 human contaminations with $\mathrm{H}_{3} \mathrm{~N}_{2} \mathrm{v}, 16$ individuals were hospitalized and one of these individuals passed on. The greater part of the individuals who were hospitalized and the individual who kicked the bucket had at least one well being or age factor that put them at high danger of genuine influenza related complexities. Individuals at high danger of genuine intricacies from occasional flu and $\mathrm{H}_{3} \mathrm{~N}_{2} \mathrm{~V}$ incorporate youngsters more youthful than 5 , individuals with certain constant conditions like asthma, diabetes, coronary illness, debilitated insusceptible frameworks, pregnant ladies and individuals 65years and more established. ${ }^{4}$

\section{Alcoholic consumption behavior and death due to swine flu}

Some demise cases because of swine influenza have the basic alcoholic utilization conduct. In light of the ongoing report on flu by Greenbaum et al.,5" 'substantial liquor utilizes expanded infection seriousness." ${ }^{5}$ Nevertheless, as of late a fascinating perception was accounted for that pneumonia related with swine influenza was increasingly normal among patients with less liquor addiction. Concentrating on casualty because of swine influenza, Cui et al. played out an examination in China and found that overwhelming liquor use was not identified with death in patients with swine influenza. Truth is told, passings because of swine influenza cases with hidden alcoholic utilization conduct are sporadically announced as case reports. The connection between alcoholic utilization conduct and seriousness/ casualty in swine influenza contamination is as yet questionable. By and by, most reports bolster no relationship. The fundamental pathologic variation from the norm in deadly cases is diffuse alveolar harm. In the creature model investigation "incessant liquor utilization may expand the danger of serious flu infection contaminations by modifying the pneumonic provocative condition and $\mathrm{CD}_{8} \mathrm{~T}$ cell reaction. Faulty invulnerable status because of ceaseless liquor abuse is accepted to be the reason for serious flu. ${ }^{6}$

Children's fear reactions to the 2009 Swine Flu pandemic: The role of threat information as provided by parents.

Research has demonstrated that dread and tension keep running in families and it appears that piece of this intra familial exchange can be credited to ecological family factors. 200 and-twenty-three kids matured 7-12years and their folks finished surveys for estimating dread convictions in connection to the Swine Flu and to what degree kids had gotten risk data about the malady from guardians and different sources. The primary outcomes demonstrate that guardians' dread degrees of the Swine Flu were fundamentally identified with youngsters' dread degree of this sickness. A critical positive relationship was found between guardians' transmission of danger data about the sickness and kids' dread levels, and this connection stayed noteworthy when controlling for different wellsprings of risk data (i.e., media, companions, and school) or direct involvement with the malady. Theory of negative data given by the guardians goes about as a middle person in the connection among guardians' and youngsters' dread of the Swine Flu. Therefore, guardians' dread of the Swine Flu was related with the transmission of risk data to their posterity, which thus was connected to youngsters' dread of the malady. ${ }^{7}$

\section{Pathology effects of swine flu virus on human patients}

Test information showed that while replication of regular flu an infection was confined to the upper aviation route, swine-starting point flu $\mathrm{A}\left(\mathrm{H}_{1} \mathrm{~N}_{1}\right)$ recreated likewise in trachea, bronchi, and bronchioles. It was shown that the 1918 flu $\left(\mathrm{H}_{1} \mathrm{~N}_{1}\right)$ infections caused serious clinical malady and necrotizing bronchiolitis and alveolitis in creatures. A 
Spanish report has uncovered that the real piece of patients with the new flu $\mathrm{A}\left(\mathrm{H}_{1} \mathrm{~N}_{1}\right)$ swine influenza had essential viral pneumonitis (90.6\%), and just one (3.1\%) had optional bacterial pneumonia. Avian flu contamination $\left(\mathrm{H}_{5} \mathrm{~N}_{1}\right)$ prompted a broad pneumonic sore with respiratory misery and diffuse alveolar harm and high death rate and the damage of the respiratory epithelium in this infection was because of an incredible arrival of cytokines. The example of cerebral injuries and nonattendance of stamped reduction of platelet check and coagulation course disturbances showed some sort of vascular sores, vascular delicacy, or even an expansion in endothelial porousness. Intense necrotizing encephalopathy is a complexity of some popular diseases - all the more habitually connected with human Herpes infection 6, flu, Para flu-which fundamentally influence little youngsters. The principle side effects of flu related with encephalopathy are seizures and adjusted cognizance. Neurological continuations are progressively visited in patient's matured $0-5$ years. ${ }^{8}$

\section{Common cold may block swine flu}

An infection that causes the normal virus might spare individuals from swine influenza. In France, influenza cases ascended toward the beginning of September, at that point remained at around 160 for each 100,000 individuals until late October, when numbers began rising once more. It was accounted for that the level of throat swabs from French respiratory ailments that tried positive for swine influenza fell in September, while simultaneously rhinovirus, which causes colds, rose. In late October, rhinovirus fell-simultaneously as influenza rose. Rhinovirus may have hindered the spread of swine influenza through a procedure called viral obstruction. This is thought to happen when one infection hinders another. It is accepted that one infection turns on the antiviral protections, and bars the different infections.

\section{Role of obesity in swine flu}

The conditions perceived as expanding the hazard from influenza are hypertension, diabetes, interminable lung impediment, coronary infection and now including weight to the rundown. It was at that point realized that stomach fat discharges a persistent stream of synthetic substances that trigger aggravation, a resistant reaction ordinarily planned for executing attacking pathogens and contaminated cells. Runaway aggravation, known as a "cytokine storm", is the thing that slaughters most influenza unfortunate casualties. In investigation scientist's estimated insusceptible synthetic compounds in the mice's blood and before disease, the corpulent mice had a lot more significant levels of a hormone called leptin than the typical mice. During the underlying phases of disease, they had less infection murdering cells and synthetic compounds. Leptin is discharged by fat cells and, in addition to other things, triggers invulnerable responses. Stout mice become desensitized to leptin, making their insusceptible framework delayed to respond. As influenza exacerbated, the stout mice mounted a resistant reaction, however it was past the point of no return. It neglected to dispose of the infection and in the end set off a runaway resistant reaction that raise until it slaughtered the mice-much as the cytokine tempest does in individuals. It is conceivable that, as in mice, heftiness hoses our capacity to battle influenza by upsetting the invulnerable reaction. ${ }^{10}$

\section{Diagnosis of swine flu virus}

Biosensors are the cutting edge analytic procedures having high affectability and particularity for pathogens. As of late, a DNA biosensor was additionally created utilizing phenyl carboxylic corrosive altered polished carbon cathode (GCE) by immobilization of DNA test and hybridization with single stranded integral DNA (sscDNA) of H1N1by estimating change in current. Silanized surface based $\mathrm{SiO}_{2}-\mathrm{IO}$ nanosensor was created which gives great surface to connection of against HA based neutralizer through natural linkers for discovery of infection by checking shift in reflectance range. The above sensor was either less delicate or tedious. The created impedimetric biosensor (HA quality explicit immobilized test) can identify as low as $0.004 \mathrm{ng}$ ss-cDNA of $\mathrm{H}_{1} \mathrm{~N}_{1}$ in $6 \mu \mathrm{L}$ just in $30 \mathrm{~min}$. The biosensor has affectability $3750 \Omega / \mathrm{cm}-2 \mathrm{ng}-1$ DNA and exceptionally explicit to $\mathrm{H}_{1} \mathrm{~N}_{1}$ infection and not different pathogens. The biosensor was likewise approved with genuine human patient examples of cDNA and corresponded with previously existing techniques, for example, RTPCR and results were discovered similar. The hemagglutinin quality based $\mathrm{H}_{1} \mathrm{~N}_{1}$ biosensor has great dependability and can be utilized in remote regions where complex instrument office doesn't exist. ${ }^{11}$

\section{Role of andrographolide from andrographis paniculata against swine flu}

Andrographis paniculata (Burm f.) Nees (Acanthaceae), is a therapeutic herb utilized in numerous locales, for example, India, Mainland China, Taiwan to treat liver issue, colic torment, normal cold, and respiratory contaminations. Andrographolide and its subordinates have been generally utilized for treating respiratory diseases in China and India for a considerable length of time. It contains diterpenoids, flavonoids and polyphenols as the major bioactive parts. Andrographolide is the major diterpenoid in A. paniculata, making up about $4 \%, 0.8 \%-1.2 \%$ and $0.5 \%-6 \%$ in dried entire plant, stem and leaf removes individually. Andrographis paniculata concentrates display antihepatotoxic, antityphoid and antifungal, antimalarial, anti-infection, antihepatitic, calming, antithrombogenic, antisnake venom properties and immunostimulating activity. Because of improvement of obstruction in numerous strains of $\mathrm{H}_{1} \mathrm{~N}_{1}$, the Food and Drug organization affirmed neuraminidase inhibitor medications, for example, Oseltamivir and Zanamivir. In an examination, the 3D structure of ACZ97474 by homology demonstrating and from that point taken for communication study among andrographolide and ACZ97474. Docking results demonstrate that andrographolide is the best among four natural mixes as its carboxyl utilitarian gathering ties with the dynamic site deposits Arg 152 alongside Lys150, and Gly197 of neuraminidase (ACZ97474) with most minimal restricting vitality $(-10.88 \mathrm{Kcal} / \mathrm{mol})$ for example high restricting effectiveness. As distinguished by docking, andrographolide was seen as most fitted ligand with ACZ97474 into dynamic site buildups than affirmed drugs for Swine influenza, for example, Zanamivir (CID:60855) and Oseltamivir (CID:65028). ${ }^{12}$

\section{Metrics and analysis}

A metric and analysis study shown in (table 1) gives information about the most frequently searched supplements in the context of $\mathrm{H}_{1} \mathrm{~N}_{1}$, with their therapeutic uses while Figure $1 \& 2$ displays the supplements with the highest probability of appearing on web pages with $\mathrm{H}_{1} \mathrm{~N}_{1}$, ranked by $P$ supplement. The public's interest in a particular therapy is indicated by two statistical analyses F supplement and P supplement. F supplement, is the frequency of hits counted separately for each supplement together with $\mathrm{H}_{1} \mathrm{~N}_{1}$. F supplement reflects the prevalence of information about supplements co-occurring with $\mathrm{H}_{1} \mathrm{~N}_{1}$ on the Internet, regardless of the context, including advertising, mentions on discussion boards or other social media, or health-related contexts. P supplement, is the proportion of total hits for a given supplement that also include $\mathrm{H}_{1} \mathrm{~N}_{1}$ on the webpage. 
Table I The most frequently searched supplements in the context of $H_{1} N_{1}$, with their therapeutic uses

\begin{tabular}{|c|c|c|}
\hline S. No. & Supplement & Use \\
\hline I. & Oscillo coccinum & Diluted extractions from duck liver and heart used for influenza. \\
\hline 2. & Tinospora & $\begin{array}{l}\text { Obtained from vine and used for high cholesterol, diabetes, allergic rhinitis, stomach } \\
\text { upset, gout, rheumatoid arthritis, lymphoma, hepatitis, peptic ulcer disease, fever, } \\
\text { syphilis, gonorrhea, immune system revitalizing. }\end{array}$ \\
\hline 3. & Elder berry extract & $\begin{array}{l}\text { Influenza treatment, HIV/AIDS, immune system revitalizing, used in sinus pain, back } \\
\text { pain, sciatica, nerve pain. }\end{array}$ \\
\hline 4. & North American ginseng & $\begin{array}{l}\text { Stress booster, immune system booster, improves digestion, general tonic, and } \\
\text { stimulant. }\end{array}$ \\
\hline 5. & Polyphenols & $\begin{array}{l}\text { Obtained from plants and includes lignins, tannins, flavonoids, possess anti oxidant } \\
\text { properties. }\end{array}$ \\
\hline 6. & Divya giloy sat & Anti-inflammatory, anti pyretic properties, immune-boosting action. \\
\hline 7. & Orange juice & Used as food product \\
\hline 8. & Echinacea & $\begin{array}{l}\text { Used in infections, such as common cold, upper respiratory infections, anti- } \\
\text { inflammatory potential, improves immunity. }\end{array}$ \\
\hline 9. & Andrographis & Used for common cold, flu, act as aorti facient \\
\hline 10. & Ban lan gen & Anti-inflammatory, Anti bacterial, anti pyretic, antiviral, and anti cancer. \\
\hline II. & Flavonoids & Anti-inflammatory properties. \\
\hline 12. & Vitamin D & Immunizer, prevent auto-immune diseases, and cancer. \\
\hline 13. & Dulcamara & $\begin{array}{l}\text { Used in itchy skin, acne, warts, broken skin, anti-arthritic, eczema, nail bed swelling, } \\
\text { diuretic, pain relief, and calming nervous excitement. }\end{array}$ \\
\hline 14. & Elecampane & Used in cough, bronchitis, asthma, nausea, worms in GI tract, Diarrhoea treatment. \\
\hline 15. & Jaggery & Used in medicine preparation for treating lung and throat infections. \\
\hline 16. & Selenium & Used in cancer prevention, heart attack, diabetes, rheumatoid arthritis. \\
\hline 17. & Mullein & Used against influenza, respiratory infections, herpes viruses. \\
\hline 18. & Eupatorium perfoliatum & Used against cancer and bacterial infections. \\
\hline
\end{tabular}

\section{Frequency of Occurrence (Fsupplement)}
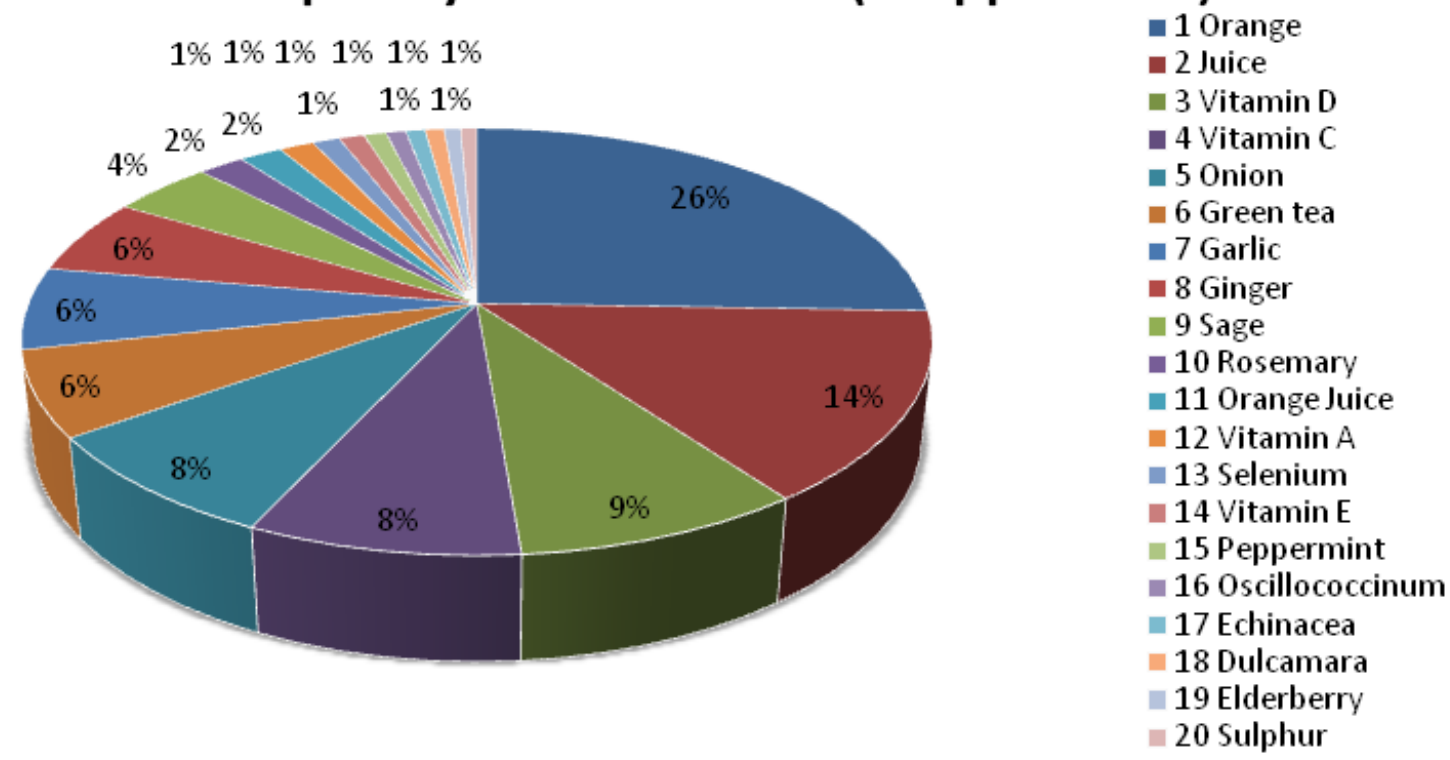

Figure I Depiction of frequency of occurrence (F supplement) of supplements together with HINI. 


\section{Probability of Co-occurence with H1N1 (Psupplement)}

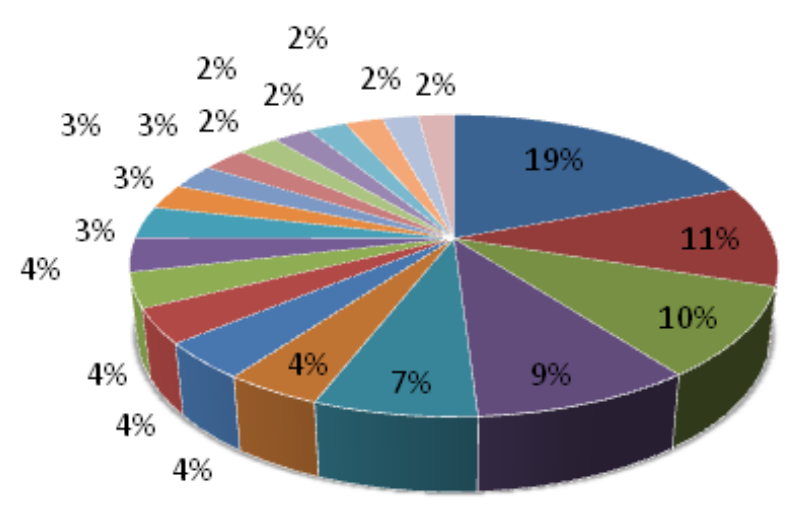

- 1 Oscillococcinum

- 2 Tinospora

3 Guduchi

- 4 Elderberry

- 5 North American ginseng

- 6 Polyphenols

- 7 Divya giloy sat

- 8 Orange Juice

m 9 Echinacea

- 10 Andrographis

- 11 Ban Lan Gen

12 Flavonoids

$=13$ Vitamin D

14 Dulcamara

15 Elecampane

n 16 Jaggery

$=17$ Selenium

$=18$ Mullein

19 Eupatorium perforliatum

20 Peppermint

Figure 2 Depiction of frequency of co-occurrence (Psupplement) of supplements together with HINI.P supplement is proportion of total hits for a given supplement that also include HINI on the webpage.

\section{Conclusion}

The Great $\mathrm{H}_{1} \mathrm{~N}_{1}$ Pandemic of 2009 is currently inclined to reunderstanding; to be anticipated into the future as a 'dress rehearsal for the genuine occasion; to leave its imprint as open door for institutional limit building. A long way from scrutinizing the job of security in human services, $\mathrm{H}_{1} \mathrm{~N}_{1}$ has fortified existing conditions of arranging and readiness. The infection showed the possibility of life where what's to come is imagined as amazement. The present investigation draws the consideration of academicians and researchers that separated from oseltamivir and other enemy of flu tranquilizes the requirement for elective wellspring of treatment of this ailment is need of this hour and researchers should concentrate on the advancement of novel meds for battling against the H1N1 viral disease. Most importantly, there is a dire requirement for worldwide coordination in clinical and epidemiologic research to control the spread of swine influenza.

\section{Acknowledgments}

None.

\section{Conflicts of interest}

The author declares that there are no conflicts of interest.

\section{Funding}

None.

\section{References}

1. https://www.webmd.com/cold-and-flu/flu-guide/h1n1-flu-virus-swineflu\#1

2. https://www.medicinenet.com/swine_flu/article.htm\#why_is_swine_ flu_now_infecting_humans
3. https://www.cdc.gov/flu/swineflu/

4. Tonks NK. Pseudophosphatases: Grab and Hold on. Cell. 2009;139(3):464-465.

5. Greenbaum A, Chaves SS, Perez A, et al. Heavy alcohol use as a risk factor for severe outcomes among adults hospitalized with laboratory confirmed influenza, 2005-2012. Infection. 2014;42(1):165-170.

6. Wiwanitkit $\mathrm{V}$. Alcoholic consumption behavior and death due to swine flu. Egyptian Journal of Chest Diseases and Tuberculosis. 2016;65:169171.

7. Remmerswaal D, Muris P. Children's fear reactions to the 2009 Swine Flu pandemic: The role of threat information as provided by parents. Journal of Anxiety Disorders. 2011;25(3):444-449.

8. Calorea EE, Uipc DE, Pereza NM. Pathology of the swine-origin influenza A (H1N1) flu. Pathology - Research and Practice. Pathology Research and Practice 2011;207(2):86-90.

9. Greer RM, McErlean P, Arden KE, et al. Do rhinoviruses reduce the probability of viral co-detection during acute respiratory tract infections? Journal of Clinical Virology. 2009;45(1):10-15.

10. https://www.newscientist.com/article/mg20327175-000-fight-the-flabto-fend-off-swine-flu/

11. Mohan RH, Gill PS, Ashok Kumar, et al. Hemagglutinin gene based biosensor for early detection of swine flu $\left(\mathrm{H}_{1} \mathrm{~N}_{1}\right)$ infection in human. Int J Biol Macromol. 2019;130(1):720-726.

12. Seniya C, Shrivastava S, Singh SK, et al. Analyzing the interaction of a herbal compound Andrographolide from Andrographis paniculata as a folklore against swine flu (H1N1). Asian Pac J Trop Dis. 2014;4(2):S624-S630. 